\title{
Cell-to-cell Fundamental Variability Limits Investigation in OxRRAM arrays
}

\author{
Alessandro Grossi, Cristian Zambelli Member, IEEE,, Piero Olivo, Etienne Nowak, \\ Gabriel Molas, Jean François Nodin, and Luca Perniola
}

\begin{abstract}
In this paper the fundamental variability limits of filament based OxRRAM are investigated considering different transistor sizes and MIM stacks featuring different materials and thicknesses. Cell-to-cell variability is analyzed through an extensive characterization of Forming, Set and Reset operations on $4 \mathrm{kbits}$ OxRRAM arrays. The results obtained in terms of switching voltage variability and resistance variability from cell to cell are compared and discussed to identify the variability limiting component as a function of the conduction regime and to understand the impact of transistor MIM stack parameters on variability and performances.
\end{abstract}

Index Terms-OxRRAM, $\mathrm{HfO}_{2}$, variability, QPC model

\section{INTRODUCTION}

Oxide-based Resistive Random Access Memories (OxRRAM) gathered increasing interest in the last few years for low-power IoT applications and neuromorphic computing [1][3] thanks to their manufacturing process simplicity, low voltage operation, and good cycling with sufficient retention capability [4], [5]. OxRRAM behavior is based on the possibility of electrically modifying the the properties of a conductive filament (CF) in a Metal-Insulator-Metal (MIM) stack: the Set operation moves the cell in a low resistive state (LRS), whereas Reset switch the cell back to a high resistive state (HRS) [6], [7]. On some technologies a preliminary Forming operation is required to activate such a switching behavior [8]-[10]. Even if several progresses were made in terms of performance and reliability in the last decade [11], variability understanding is still the major technical roadblock for OxRRAM widespread adoption. Recently, the origin of the resistance spread was determined [12] and the fundamental variability limits of filamentary-based OxRRAM were identified, showing that the main limiting components in $1 \mathrm{~T}-1 \mathrm{R}$ architectures are: the select transistor when CF is formed or set to a low resistive state (LRS), and the MIM stack properties when CF is brought to a high resistive state (HRS) [13], [14]. In this work, the boundaries of such limits are explored by performing an extensive study on 4kbits OxRRAM arrays featuring different select transistor geometries and MIM stacks with different materials and thicknesses. Cell-to-cell variability is analyzed and compared during Forming, Set and Reset, in attempt to confirm the universal nature of those limits

A. Grossi, C. Zambelli and P. Olivo are with Dipartimento di Ingegneria Università degli Studi di Ferrara, Via Saragat 1, Ferrara (Italy), 44122 (e-mail: alessandro.grossi@unife.it).

E. Nowak, G. Molas, J. F. Nodin, and L. Perniola are with CEA-Leti, Minatec Campus, Grenoble, France

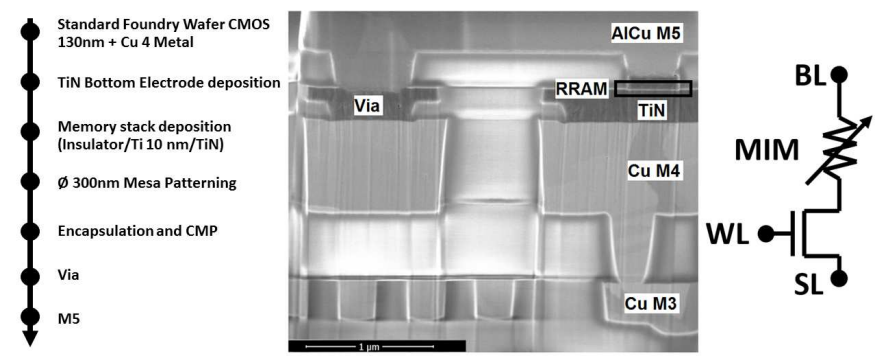

Fig. 1. Description of the integration flow, TEM cross section of the integrated TiN/HfO 2 /Ti/TiN OxRRAM [14] and 1T-1R cell schematic.

while understanding the impact of the 1T-1R technological parameters on variability and switching capabilities.

\section{DeVices Fabrication}

The device samples of this work base on a OxRRAM technology integrated on 130nm CMOS logic. On top of $\mathrm{Cu}$ Metal 4, a TiN bottom electrode is defined. Then a Insulator/Ti $10 \mathrm{~nm} / \mathrm{TiN}$ stack is deposited. Main integration steps and cross section of a $300 \mathrm{~nm}$ diameter integrated device are described in Fig. 1(left). The current pristine state of the cell has been measured around $1 \mathrm{pA}$ from $1 \mathrm{R}$ structure applying $100 \mathrm{mV}$ on top electrode.

\section{EXPERIMENTAL SETUP}

The electrical measurements were performed on 4kbits 1T$1 \mathrm{R}$ arrays where the bitline (BL) voltage defines the voltage applied on the top electrode, the sourceline (SL) defines the voltage applied on the bottom electrode and the wordline (WL) voltage sets the compliance current $\left(\mathrm{I}_{C C}\right)$ during the operations on the 1T-1R cells as shown in Fig. 1(right). 4 kbits 1T-1R array measurement shows that the high resistive state (HRS) and low resistive state (LRS) can be separated up to $3 \sigma$ with no extrinsic bits. The setup is divided in two parts. The analogic part is constituted by a Keysight B1530 which allows to apply pulsed voltages during Forming, Set, Reset and Read operations. It also allows to read currents with a resolution of 1fA. The digital addressing part, based on an Arduino microcontroller, drives the MUXs allowing to select sequentially the cells in the array.

\section{EXPERIMENTAL RESULTS}

\section{A. Forming operation}

As already demonstrated in [14], Forming operations performed with a low compliance current allow forming a cells 
population in HRS, reducing the power consumption during Forming. After Forming in HRS, cells can be switched to LRS with a single pulse without inducing any performance degradation. The cumulative distribution of the time required to activate the Forming process when a constant voltage stress is applied, causing the conductive filament activation, can be modeled as a Weibull distribution. To prove that assumption on the different MIM stacks described in Tab. I, we performed Forming operation by using a sequence of pulses [14] with length of $100 \mathrm{~ns}$, bitline (BL) voltage of $4 \mathrm{~V}$ and $\mathrm{I}_{C C}=5 \mu \mathrm{A}$. When $3 \sigma$ range of the cells population in the $4 \mathrm{kbits}$ array (i.e., corresponding to $99.7 \%$ of the population) was formed, the pulse train operation was stopped. We observed that in all cases the formed cells percentage as a function of the forming time statistically follows a Weibull law, whose characteristic parameters (i.e., the scale factor $\eta$ and the form factor $\beta$ ) vary with the MIM stack technological parameters (i.e., constituting materials and thickness of the insulator). The cumulative distribution function of the Weibull distribution is expressed with the following well-known equation:

$$
F(t)=1-e^{-(t / \eta)^{\beta}}
$$

The distribution parameters associated with each MIM stack are exposed in Tab. II. The formed cells percentage calculated during pulse sequence Forming and the Weibull fittings are shown in Fig. 2. A large forming time variability is observed in all MIM stacks: in order to reduce the power consumption during Forming, the use of a program and verify will be beneficial [15]. When comparing stacks with same materials but different thickness such as A with B or C with $\mathrm{D}$, we can observe that a thickness reduction of the insulator material generally causes a variability, forming cumulative time and energy (calculated as the product between forming voltage,current and time) reduction, resulting in an increase of both $\eta$ and $\beta$ fitting parameters. An increase of the Weibull slope parameter $\beta$ is related to the decrease of the median resistance measured after Forming, corresponding to a decrease of the overall cell-to-cell variability [16]. When comparing different MIM stacks, it is shown that those with pure $\mathrm{HfO}_{2}$ insulator $(\mathrm{A}, \mathrm{B})$ require a shorter cumulative forming time compared to the stacks with an additional $1 \mathrm{~nm} \mathrm{Al}_{2} \mathrm{O}_{3}$ layer $(\mathrm{C}, \mathrm{D})$ or Al-doped insulator (E). This is ascribed to a reduced atomic mobility in $\mathrm{Al}_{2} \mathrm{O}_{3}$ stacks and Al-doped insulators, causing an increase of the energy required in forming [17]. The results do not show a predictive trend for short pulse length even though the thinner dielectric required a shorter cumulative forming time than the ticker one. This unexpected behavior could be related to pristine resistance variability and to the fact that the forming was done with a low compliance current, which brings the cells in an intermediate state between LRS and HRS.

We already observed in [14] that the cell-to-cell variability during Forming is directly related to the median resistance, hence it is possible to fit the relationship between the standard deviation of the measured resistance from the cells population and the median resistance with the universal law defined in [14] as:
TABLE I

MIM STACKS DESCRIPTION

\begin{tabular}{|l|c|c|}
\hline Process & Insulator & thickness \\
\hline $\mathrm{A}$ & $\mathrm{HfO}_{2}$ & $10 \mathrm{~nm}$ \\
$\mathrm{~B}$ & $\mathrm{HfO}_{2}$ & $5 \mathrm{~nm}$ \\
$\mathrm{C}$ & $\mathrm{Al}_{2} \mathrm{O}_{3} / \mathrm{HfO}_{2}$ & $1 \mathrm{~nm} / 10 \mathrm{~nm}$ \\
$\mathrm{D}$ & $\mathrm{Al}_{2} \mathrm{O}_{3} / \mathrm{HfO}_{2}$ & $1 \mathrm{~nm} / 5 \mathrm{~nm}$ \\
E & $\mathrm{HfAlO}$ & $10 \mathrm{~nm}$ \\
\hline
\end{tabular}

TABLE II

FORMING TIME, ENERGY AND WEIBULL FITTING PARAMETERS

\begin{tabular}{|l|c|c|c|c|}
\hline Process & $3 \sigma$ Forming time & $3 \sigma$ Energy & $\eta$ & $\beta$ \\
\hline A & $40 \mu \mathrm{s}$ & $0.8 \mathrm{~nJ}$ & $288 \mathrm{~ns}$ & 0.35 \\
B & $10 \mu \mathrm{s}$ & $0.2 \mathrm{~nJ}$ & $670 \mathrm{~ns}$ & 0.65 \\
$\mathrm{C}$ & $180 \mu \mathrm{s}$ & $3.6 \mathrm{~nJ}$ & $26.5 \mu \mathrm{s}$ & 0.91 \\
$\mathrm{D}$ & $130 \mu \mathrm{s}$ & $2.6 \mathrm{~nJ}$ & $45 \mu \mathrm{s}$ & 1.68 \\
$\mathrm{E}$ & $80 \mu \mathrm{s}$ & $1.6 \mathrm{~nJ}$ & $12.9 \mu \mathrm{s}$ & 0.97 \\
\hline
\end{tabular}

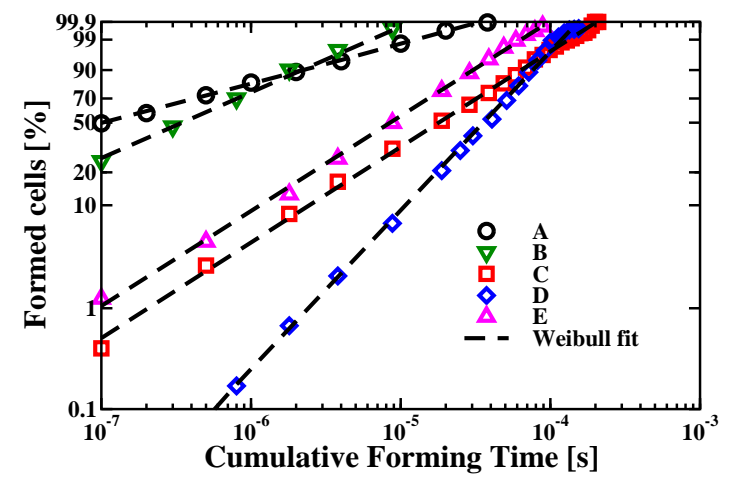

Fig. 2. Formed cells percentage during pulse train Forming.

$$
\sigma=\sqrt{G_{0} e^{-\alpha \phi}} R^{1.5}
$$

where $\mathrm{G}_{0}=(12.9 \mathrm{k} \Omega)^{-1}$ is the quantum conductance unit corresponding to the creation of a single conductive nanowire. According to Quantum Point Contact (QPC) model [18], [19], the conductive filament interruption after Reset can be modeled with the introduction of a potential barrier at bottom of the first quantized level, whose modeling parameters are $\phi$ (i.e., the barrier height) and $\alpha$ (related to the width of the barrier, assuming the presence of a parabolic longitudinal potential). The relationship among $\alpha \phi$ and variability was investigated in [14]. Fig. 3 shows the cumulative distributions of the cell resistances and $\sigma$ versus median $\mathrm{R}$ characteristics obtained after Forming: we see that even if the resistance distributions differ to each other, the $\sigma$ versus median $\mathrm{R}$ relationship follows Eq.(2) with $\alpha \phi=2$ independently from the MIM stack. Changing MIM stack material and thickness has an impact on the time and energy required to perform the Forming operation, on the variability of such parameters and on the median resistance obtained after Forming: this is in agreement with previous results [20], [21] in which the relationship among resistance compliance current, film materials and thickness are investigated. However, the relationship between median resistance and cell-to-cell variability is maintained for all MIM stacks independently from the MIM materials and thickness. 


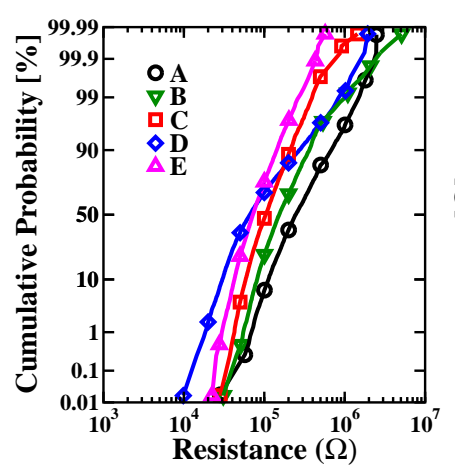

(a)

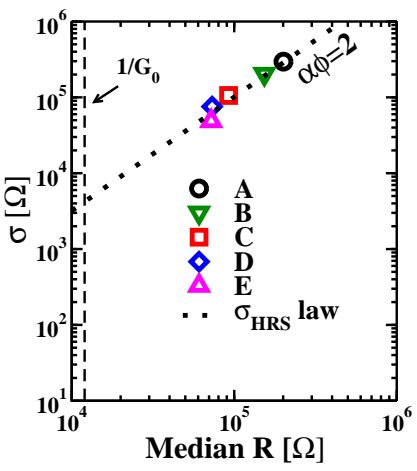

(b)
Fig. 3. Cumulative distribution of read resistance obtained after Forming (a) $\sigma$ versus median $\mathbf{R}$ plot obtained after Forming.

\section{B. Set/Reset operations}

After Forming, the switching and cell-to-cell variability features were compared. To this extent, several single pulse Set and Reset conditions were considered by varying BL, SL voltages and compliance current to explore the full resistance range between $1 \mathrm{k} \Omega$ and $1 \mathrm{M} \Omega$. The $\mathrm{BL}$ voltage in Set and SL voltage in Reset were varied from 0 to $3.5 \mathrm{~V}$ with steps of $0.1 \mathrm{~V}$ while keeping $\mathrm{I}_{C C \text {, set }}=0.4 \mathrm{~mA}$ and $\mathrm{I}_{C C \text {, reset }}=2.2 \mathrm{~mA}$. After that, on newly formed arrays the compliance current in Set was varied from $0.2 \mathrm{~mA}$ to $0.9 \mathrm{~mA}$ with $\mathrm{V}_{B L}=2 \mathrm{~V}$ and in Reset from $2 \mathrm{~mA}$ to $4 \mathrm{~mA}$ with $\mathrm{V}_{S L}=2.5 \mathrm{~V}$. In Fig. 4(a), average Set and Reset BL and SL voltages required to obtain a read resistance of $6 \mathrm{k} \Omega$ after Set and $30 \mathrm{k} \Omega$ after Reset are reported with $\mathrm{T}_{P U L S E}=100 \mathrm{~ns}, \mathrm{I}_{C C, \text { set }}=0.4 \mathrm{~mA}$ and $\mathrm{I}_{C C \text {, reset }}=2.2 \mathrm{~mA}$, respectively. Minimum and maximum voltages required to switch up to $95.4 \%$ of the cells in the array are shown. When considering the $\mathrm{HfO}_{2}$-based MIM stacks, a thickness reduction allows to decrease the Set voltage average value and variability whereas the Reset voltage average value and variability increase. The same effect is observed on Set when the $\mathrm{Al}_{2} \mathrm{O}_{3} / \mathrm{HfO}_{2}$ stacks are considered. However, in this latter case, the thickness reduction has positive effects also on Reset since it reduces both its average voltage and variability. When comparing different stacks with same thickness (A,C,E), process $\mathrm{C}$ shows the lowest Set switching voltage and variability, whereas process A shows the lowest Reset switching voltage and variability. Fig. 4(b) shows $\sigma$ versus median R characteristics obtained while varying the $\mathrm{I}_{C C}$ during Set from $0.2 \mathrm{~mA}$ up to $0.9 \mathrm{~mA}$ with steps of $0.1 \mathrm{~mA}$ and $\mathrm{V}_{B L}=2 \mathrm{~V}$, and during Reset from $2 \mathrm{~mA}$ to $4 \mathrm{~mA}$ with steps of $0.2 \mathrm{~mA}$ and $\mathrm{V}_{S L}=2.5 \mathrm{~V}$ to explore the full resistance range. As previously observed in Forming, the variability in HRS always follow Eq.(2) with $\alpha \phi$ between 2 and 3.2 when the resistance is above $1 / \mathrm{G}_{0}$ whereas it goes under the limit below $1 / \mathrm{G}_{0}$ in all cases: this confirms that changing insulator in the MIM stack has an impact on the switching voltages and their variability whereas the $\sigma$ versus median $\mathrm{R}$ values above $1 / \mathrm{G}_{0}$ only depends on the median resistance for all filamentary based OxRRAM. Even if this was already demonstrated in [14] for standard $\mathrm{HfO}_{2}$ OxRRAM, this analysis allows to extend the validity of the relationship to MIM stacks featuring different materials and

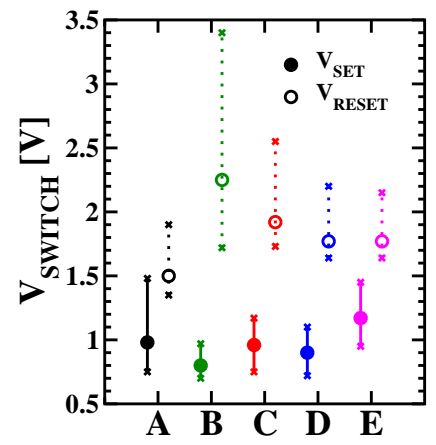

(a)

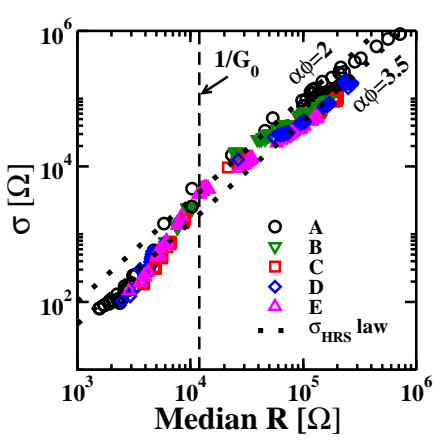

(b)
Fig. 4. Average Set (BL) and Reset (SL) switching voltages and their standard deviations (a). Resistance distributions after Forming (a) and $\sigma$ versus median $\mathrm{R}$ plot after Forming (b).

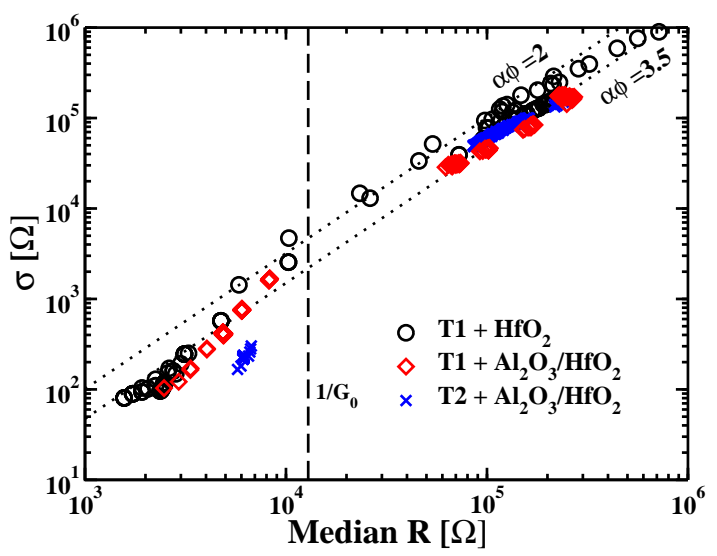

Fig. 5. $\sigma$ versus median $\mathrm{R}$ plot evidencing the role of the select transistor size on the variability.

thicknesses. In conclusion, the choice of the insulator material will impact the time and power consumption during Forming, Set and Reset but not the cell-to-cell variability, which can be limited only at circuit and system level by using program with verify algorithms and ECC [15].

\section{Select transistor impact}

To evaluate the impact of the transistor in LRS variability, the same analysis was performed on the MIM stack of process $\mathrm{C}$ integrating two transistors with different sizes. Fig. 5 shows a comparison of the $\sigma$ versus median R relationship obtained considering two processes with the same transistor (T1) but different MIM stack $(\mathrm{B}, \mathrm{C})$ and process $\mathrm{C}$ with a smaller transistor (T2). While no variation is observed in $\sigma$ versus median $\mathrm{R}$ values above $1 / \mathrm{G}_{0}$, below the limit a different impact is observed with the smaller transistor T2, confirming that it defines the variability in such region. When $\mathrm{T} 2$ is considered, a lower compliance current is used in during both Set $\mathrm{I}_{C C, \text { set }}=0.2 \mathrm{~mA}$ and Read operations, resulting in lower noise and lower variability of the measured resistance values. Moreover, the use of smaller transistor with lower compliance current will be beneficial in terms of power consumption. 


\section{CONCLUSiOnS}

The cell-to-cell variability during Forming, Set and Reset operations is compared considering different MIM stacks and transistors in 4kbits OxRRAM arrays. The experimental results confirmed that, when single pulse operations are considered, above $1 / \mathrm{G}_{0}$ the $\sigma$ versus median $\mathrm{R}$ characteristics is not dependent from the MIM stack material, thickness and select transistor but only depends on the median read resistance. The MIM stacks materials and thickness only influence the switching voltages, their variability and the median resistance obtained after switching. Below $1 / \mathrm{G}_{0}$, on the other hand, the cell-to-cell variability depends on the transistor.

\section{REFERENCES}

[1] J. F. Kang, B. Gao, B. Chen, L. F. Liu, X. Y. Liu, H. Y. Yu, Z. R. Wang, and B. Yu, "Oxide-based rram: A novel defect-engineeringbased implementation for multilevel data storage," in IEEE Int. Memory Workshop, May 2012, pp. 1-4.

[2] T. Werner, E. Vianello, O. Bichler, A. Grossi, E. Nowak, J. F. Nodin, B. Yvert, B. DeSalvo, and L. Perniola, "Experimental demonstration of short and long term synaptic plasticity using oxram multi k-bit arrays for reliable detection in highly noisy input data," in IEEE Int. Electron Devices Meeting (IEDM), Dec 2016, pp. 16.6.1-16.6.4.

[3] M. Ueki, K. T. Akeuchi, T. Yamamoto, A. Tanabe, N. Ikarashi, M. Saitoh, T. Nagumo, H. Sunamura, M. Narihiro, K. Uejima, K. Masuzaki, N. Furutake, S. Saito, Y. Yabe, A. Mitsuiki, K. Takeda, T. Hase, and Y. Hayashi, "Low-power embedded ReRAM technology for IoT applications," in IEEE Symposium on VLSI Circuits, June 2015, pp. T108-T109.

[4] S. F. Karg et al., "Transition-metal-oxide-based resistance-change memories," IBM Journal of Research and Development, vol. 52, no. 4.5, pp. 481-492, 2008.

[5] R. Waser, Nanoelectronics and Information Technology: Advanced Electronic Materials and Novel Devices. New York, NY, USA: John Wiley \& Sons, Inc., 2003.

[6] G. Bersuker et al., "Metal oxide resistive memory switching mechanism based on conductive filament properties," Journal of Applied Physics, vol. 110 , no. 12 , pp. $1-12,2011$

[7] S. Yu, X. Guan, and H.-S. Wong, "On the switching parameter variation of metal oxide RRAM; part ii: Model corroboration and device design strategy," IEEE Trans. on Electron Devices, vol. 59, no. 4, pp. 1183 1188, 2012.

[8] P. Lorenzi, R. Rao, and F. Irrera, "Forming kinetics in $\mathrm{HfO}_{2}$-based RRAM cells," IEEE Trans. on Electron Devices, vol. 60, no. 1, pp. 438-443, 2013.

[9] A. Grossi et al., "Impact of intercell and intracell variability on forming and switching parameters in RRAM arrays," IEEE Trans. on Electron Devices, vol. 62, no. 8, pp. 2502-2509, 2015.

[10] A. Kalantarian et al., "Controlling uniformity of RRAM characteristics through the forming process," in IEEE Int. Reliability Physics Symposium (IRPS), April 2012, pp. 6C.4.1-6C.4.5.

[11] L. Perniola, G. Molas, G. Navarro, E. Nowak, V. Sousa, E. Vianello, and B. D. Salvo, "Universal Signatures from Non-Universal Memories: Clues for the Future..." in IEEE Int. Memory Workshop (IMW), May 2016, pp. 1-3.

[12] J. Guy, G. Molas, C. Cagli, M. Bernard, A. Roule, C. Carabasse, A. Toffoli, F. Clermidy, B. D. Salvo, and L. Perniola, "Guidance to reliability improvement in CBRAM using advanced KMC modelling,' in IEEE Int. Reliability Physics Symposium, May 2017, pp. 1-4.

[13] S. Ambrogio, S. Balatti, A. Cubeta, A. Calderoni, N. Ramaswamy, and D. Ielmini, "Statistical fluctuations in hfox resistive-switching memory: Part i - set/reset variability," IEEE Trans. on Electron Devices, vol. 61, no. 8, pp. 2912-2919, Aug 2014.

[14] A.Grossi, E. Nowak, C. Zambelli, C. Pellissier, S. Bernasconi, G. Cibrario, K. E. Hajjam, R. Crochemore, J. Nodin, P. Olivo, and L. Perniola, "Fundamental Variability Limits of Filament-based RRAM," in IEEE Int. Electron Devices Meeting (IEDM), Dec 2016, pp. 4.7.14.7.4.
[15] A. Hayakawa, K. Maeda, S. Fukuyama, H. Takishita, R. Yasuhara, S. Mishima, and K. Takeuchi, "Resolving Endurance and Program Time Trade-off of 40nm TaOx-based ReRAM by Co-optimizing Verify Cycles, Reset Voltage and ECC Strength," in IEEE Int. Memory Workshop, May 2017, pp. 1-4.

[16] M. Zhang, S. Long, Y. Li, Q. Liu, H. Lv, and M. Liu, "Current compliance impact on the variability of hfo2-based rram devices," in IEEE Silicon Nanoelectronics Workshop (SNW), June 2016, pp. 96-97.

[17] B. Traoré, P. Blaise, E. Vianello, H. Grampeix, S. Jeannot, L. Perniola B. D. Salvo, and Y. Nishi, "On the origin of low-resistance state retention failure in hfo2-based rram and impact of doping/alloying," IEEE Tran. on Electron Devices, vol. 62, no. 12, pp. 4029-4036, Dec 2015.

[18] E. A. Miranda, C. Walczyk, C. Wenger, and T. Schroeder, "Model for the Resistive Switching Effect in $\mathrm{HfO}_{2}$ MIM Structures Based on the Transmission Properties of Narrow Constrictions," IEEE Electron Device Letters, vol. 31, no. 6, pp. 609-611, 2010.

[19] E. Miranda, D. Jimenez, and J. Sune, "The quantum point-contact memristor," IEEE Electron Device Letters, vol. 33, no. 10, pp. 1474 1476, 2012.

[20] E. Vianello, O. Thomas, G. Molas, O. Turkyilmaz, N. Jovanović, D. Garbin, G. Palma, M. Alayan, C. Nguyen, J. Coignus, B. Giraud, T. Benoist, M. Reyboz, A. Toffoli, C. Charpin, F. Clermidy, and L. Perniola, "Resistive memories for ultra-low-power embedded computing design," in 2014 IEEE International Electron Devices Meeting, Dec 2014, pp. 6.3.1-6.3.4.

[21] D. Ielmini, "Modeling the universal set/reset characteristics of bipolar rram by field- and temperature-driven filament growth," IEEE Transactions on Electron Devices, vol. 58, no. 12, pp. 4309-4317, Dec 2011. 